\title{
An Overview of Knowledge Management Techniques for e-Recruitment
}

\author{
Jorge Martinez-Gil
}

\begin{abstract}
The number of potential job candidates, and therefore costs associated to their hiring, has grown significantly in the recent years. This is mainly due to both the complicated situation of the labor market and the increased geographical flexibility of employees. Some initiatives for making the e-Recruitment processes more efficient have notably improved the situation by developing automatic solutions. But there are still some challenges that remain open since traditional solutions do not consider semantic relations properly. This problem can be appropriately addressed by means of a sub discipline of knowledge management called semantic processing. Therefore, we overview the major techniques from this field that can play a key role in the design of a novel business model that is more attractive for job applicants and job providers.
\end{abstract}

Keywords: Knowledge Management, Human Resource Management, eRecruitment

\section{Introduction}

In the field of Human Resource Management (HRM), one of the most important tasks consists of recruiting new employees (Malinowski et al., 2006). The importance of this task is due to the fact employees are the skilled players contributing to the achievement of the strategic goals of the organization they work for. Therefore, choosing and hiring new employees from a wide and heterogeneous range of candidates is of vital importance for the future success of the organizations which hire them. 
One of the major problems in this scenario is that due to the complicated situation of the labor market in many countries of the world and the increased geographical flexibility of employees, employers often receive a huge number of applications for an open position. This means that the costs of manually selecting potential candidates may rise. In this way, most employers want to decrease transaction costs when publishing job postings and selecting appropriate applicants from such a plethora of potential candidates (Bizer et al., 2005). On the other side, unsuccessful job applicants often complain on the lack of transparency in their search for a position, and they often wish to receive a detailed explanation, or at least, some feedback about the flaws of their profiles. However, they do not receive any kind of feedback very often since this has to be done manually, and it is quite expensive for the companies to do that.

These problems can be addressed by means of an automatic matching process between applicant profiles and job offers. This solution is good for employers which can make the recruitment process may become cheaper, faster and more successful, but also for job applicants who can receive informative feedback about the recruitment decisions concerning their applications. Tackling this goal of such a win-win situation can be done by using some knowledge management techniques combined with background knowledge about the Human Resources (HR) domain. This background knowledge can be stored and refined in a HRKnowledge Base (Martinez-Cruz et al., 2012). In this way, it is possible not only to identify automatically the best candidate, but also to elaborate a ranking containing the most promising ones. And not least important, automatically providing feedback to all job applicants concerning the status of their applications and detailed reasons for hiring or rejecting them is also possible. Moreover, this functionality represents an added value service that companies can offer without any additional cost for them.

Knowledge management is a broad discipline covering many aspects concerning the use of explicit knowledge for solving real problems by means of computers. One subfield of this discipline, semantic processing (Wen et al., 2012), fits well in the HR scenario. The reason is that techniques for semantic processing can be used for understanding beyond the literal lexical matching of words by analyzing their meanings at the conceptual level. In this way, when analyzing the 
curriculum of job candidates, this kind of techniques can operate at the conceptual level when comparing specific terms (e.g., Finance) also could yield matches on related terms (e.g., Economics, Economic Affairs, Financial Affairs, etc.). As another example, in the healthcare field, an expert on the treatment of cancer could also be considered as an expert on oncology, lymphoma or tumor treatment, etc. The potential of this kind of techniques is that it can support HRM when leading to a more quickly and easily cut through massive volumes of potential candidate information.

The overall goal of this overview consists of describing advances in eRecruitment through the use of semantic processing techniques. This is particular relevant since using these techniques can lead to a number of substantial improvements over the state-of-the-art concerning job recruitment processes in HRM systems. Moreover, appropriately addressing this problem has a strong exploitation potential for the HR industry due to the fact that current computational solutions for candidate profile and job description matching need to deliver more accurate results.

The rest of this paper is structured as follows: Section 2 describes the current state-of-the-art concerning advanced systems for automation of recruitment processes. Section 3 the Problem Statement concerning e-Recruitment and explains why some advanced knowledge management techniques can help to overcome many of the current challenges in this field. Section 4 describes the scientific foundations in which HRM systems using knowledge management are based. Finally, we remark the conclusions and put forward future lines of research in this field.

\section{Related Work}

The problem of automatically matching job offers and applicant profiles is not new and has been studied in the scientific literature (Färber et al., 2003) but the complex nature of the problem, which involves the use of free text by employers (when writing their job offers), and by employees (when writing their curriculums), makes developed solutions cannot reach a high degree of success. Some works have offered partial solutions based on the use of controlled vocabularies in order 
to fairly alleviate some problems concerning semantic heterogeneity (Colucci et al., 2003) but there are still some key challenges that should be addressed. In fact, last years have been even more intense in terms of research on new eRecruitment techniques. This is mainly due to the needs for computer-based intelligent techniques for recruiting employees in a highly competitive global market have grown significantly during the last times.

A number of works have detected the need of smarter e-recruitment systems for making the recruitment process more effective and efficient. Most of them agree with us to point that some kind of explicit knowledge could help to address this challenge. For instance,

Faliagka et al. (Faliagka et al., 2012) present an approach for recruiting and ranking job applicants in online recruitment systems, with the objective to automate applicant pre-screening. The applicant's rank is derived from individual selection criteria using an analytical hierarchy process, while their relative significance is controlled by the recruiter. This is also the first work that includes automated extraction of candidate personality traits using linguistic analysis.

Kumaran and Sankar (Kumaran \& Sankar, 2013) present EXPERT; a system which has three phases in screening candidates for recruitment. In a first phase, the system collects candidate profiles and constructs an ontology document for the features of the candidates. Job requirements are represented as ontology in the second phase and in the third phase, EXPERT maps the job requirement ontology into the candidate ontology document and retrieves the eligible candidates.

Daramola et al. (Daramola et al., 2010) describe the implementation of a fuzzy expert system (FES) for selecting qualified job applicants with the aim of minimizing the rigor and subjectivity associated with the candidate selection process. The novelty of this approach consists of handling the fuzziness that is associated with the problem of personnel recruitment. 
Garcia-Sanchez et al. (Garcia-Sanchez et al., 2006) present a system where the knowledge of the recruitment domain has been represented by means of ontology. This ontology is used to guide the design of the application and to supply the system with semantic capabilities. Furthermore, the ontological component allows defining an ontology-guided search engine which provides more intelligent matches between job offers and candidates profiles.

Bradley and Smyth (Bradley \& Smith, 2003) present CASPER, an online recruitment search engine, which attempts to address this issue by extending traditional search techniques with a personalization technique that is capable of taking account of user preferences as a means of classifying retrieved results as relevant or irrelevant

Finally, Khosla et al. (Khosla et al., 2009) present ISRBS; a tool for representing the findings and outcomes based on field studies and random surveys of salespersons as well as development of models for measuring independent and dependent variables related to selling behavior.

Within this overview, we aim to describe advances in e-Recruitment through the use of semantic processing techniques. Despite of many of the surveyed works have touched to some extent one or more aspects of semantic processing, there is not any study offering an overall view about the benefits of semantic matching when designing, building and exploiting advanced systems for automation of recruitment processes.

\section{Problem Statement}

Semantic matching is a field of research whereby two objects (whatever the nature of these objects) are assigned a score based on the likeness of their meaning. Let us suppose that these objects are texts representing applicant profiles and job offers; if these texts present a kind of structure then the matching process can be even more accurate since it is possible to get profit from additional information about the structure of these applicant profiles and job offers. Semantic matching is considered to be one of the pillars for many 
computer related fields since a wide variety of techniques, such as clustering, data matching, data mining or machine translation rely on a good performance when determining the meaning of data they work with.

In Figure 1, we can see a common situation where we have a job offer and applicant profile represented by means of two lattices. The job offer tells us that a given company is looking for a person who has a Bachelor degree in Finance, who masters Data Mining and the $\mathrm{C}_{++}$programming language, and is a good team worker and communicator. The job applicant has two Bachelor degrees (Economics and Computer Science), is skilled in the field of Time Series Analysis and the Java language, and finally is a good team worker and an analytical thinker. Now, one algorithm should determine the fitness of this candidate for the job offer automatically. Let us suppose that there is not a problem of semantic heterogeneity since we are working with a cutting-edge recruitment system, and therefore, both offer and profile have been written using a controlled vocabulary.
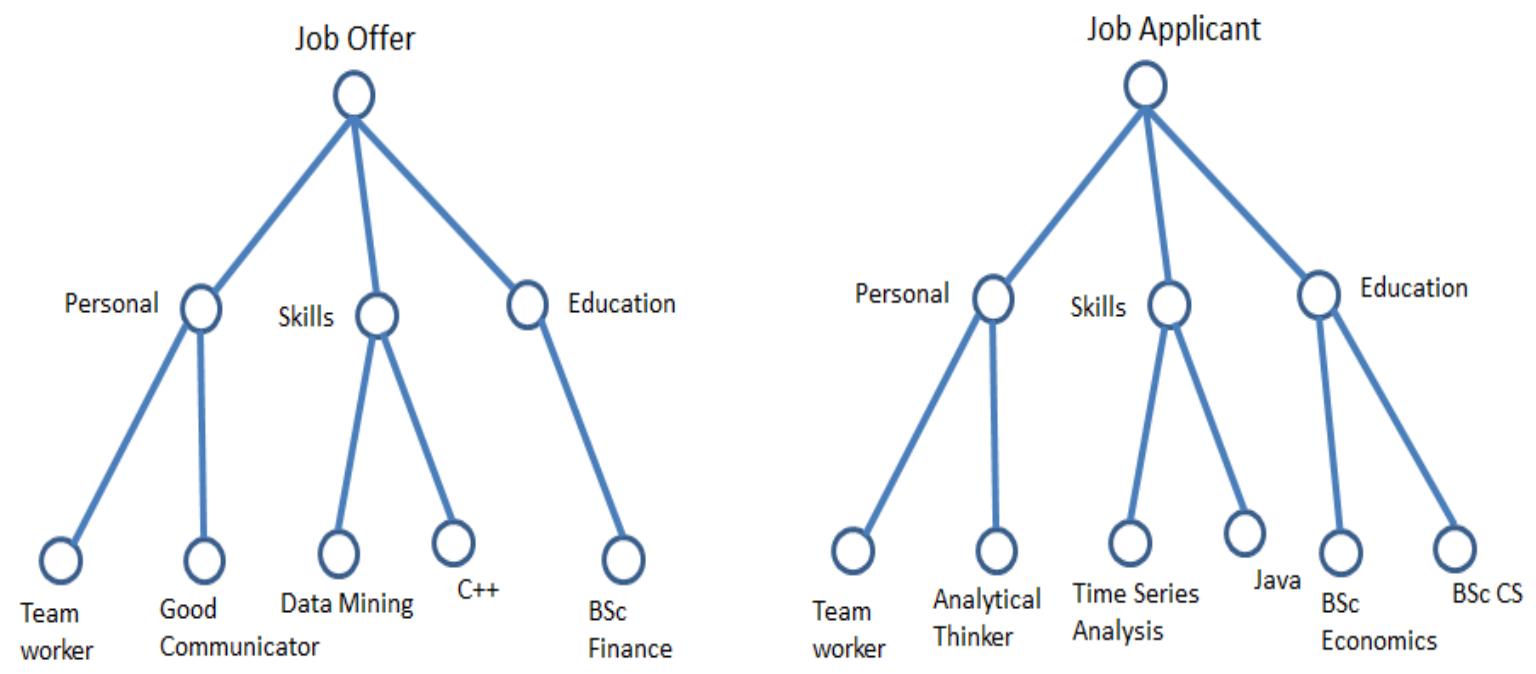

Figure 1. Matching scenario where an applicant profile should receive a fitness concerning its suitability for a specific job offer. In this case, job offer and job applicant have an overlapping node (Team worker), therefore the fitness score would be $1 / 5(0.2)$

According to the traditional way to proceed, and since we have used a controlled vocabulary, a computational algorithm should look for the number of overlapping nodes in the two lattices. In this case, job offer and job applicant have only one overlapping node (Team worker). This means that of five requirements for the offer only one is satisfied. As a result, we have that the fitness score for the given 
job applicant concerning this job offer would be $1 / 5(0.2)$ which is a score that does not reflect the semantic relations properly.

Semantic matching provides a more sophisticated way to solve this kind of problem. It is obvious that there is only one overlapping node, but our HRKnowledge Base may contain some information stating that $\mathrm{C}_{++}$and Java programming languages are two similar Object-Oriented computational programming languages, so that our algorithm may grant some extra score to the overall fitness. Moreover, our HR-Knowledge Base may state that a Bachelor in Finance is related (to some extent) to a Bachelor in Economics, so it has sense to add some extra points to the overall score too. It should also be possible that our HR-Knowledge Base may state that there are incompatible skills or personal attitudes between the applicant and the offer. In this case, a penalty could be considered. Therefore, the overall fitness is more complex to compute, but it is also much more sophisticated than the traditional one. This is mainly due to the fact that semantic aspects are being considered in the way a human expert would do that, and even better, since the HR-Knowledge Base can contain vast amounts of specific knowledge. This way to proceed gives more opportunities to the good candidates, but also allows companies to identify the talent which otherwise may remain hidden.

\section{Scientific Foundations}

The key of success when using knowledge management for e-Recruiting is the appropriate exploitation of HR-Knowledge Bases making use of declarative knowledge about specific domains, so that some recruiting processes can be cheaper, faster, more accurate and reflect the way human experts take decisions in the HR domain. Moreover, within natural language processing, information extraction or retrieval, computational systems can profit from knowledge bases to provide information at different levels of detail.

On the other hand, it is well known that most of knowledge-based systems suffer from the so called knowledge acquisition bottleneck, that is to say, it is difficult to model the knowledge relevant for the domain in question (Cimiano et al., 2004). 
Therefore, this kind of development is known to be a hard and time-consuming task. For this reason, there are some proposals to design and develop new computational methods for automatic knowledge base learning which can automate this task.

Figure 2 shows a conceptual representation of a HRM using knowledge management. This means there are a number of objects including job offers which are written using some kind of controlled vocabulary, a database of applicant's profiles which have been also written using a controlled vocabulary, a HR-Knowledge Base which contains a lattice modeling concepts, attributes for these concepts, and relationships between the concepts, and also reports containing useful statistics about the job applicants. The key challenges in this field are a) the matching process which consists of automatically computing the fitness for each applicant profile concerning a job offer, b) the improvement of the matching process by learning from past solved cases, c) the enrichment process which consists of adding new knowledge (extracted from the database of job applicants) to the HR-Knowledge Base, and d) designing an improved querying process which consists of getting useful statistics from the database of applicant's profiles.

The role of the HR-Knowledge Base is of vital importance in this kind of approaches since it is a knowledge repository that provides a great valuable support for the matching and query processes. Related to matching, explicit knowledge about a specific industrial domain helps to identify the degree of affinity between skills, competencies or personal skills. Concerning to querying, knowledge helps to formulate more complex requests which do not need a perfect (but an approximate) match in a reasonable response time. 


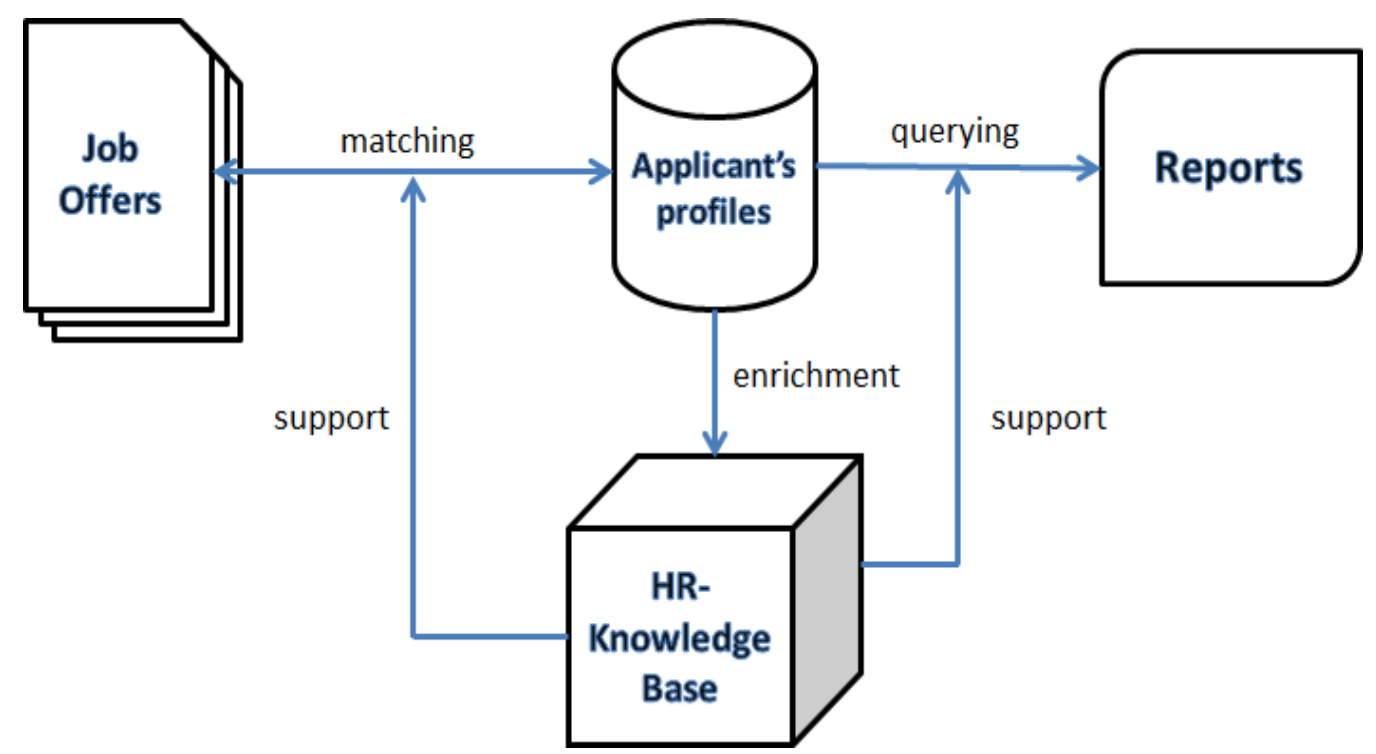

Figure 2. The HR-Knowledge Base is intended to serve as a knowledge repository to support for the matching and query processes. Related to matching, explicit knowledge about a specific industrial domain helps to identify the degree of affinity between skills, competencies or personal skills. Concerning to querying, knowledge helps to formulate more complex requests which do not need a perfect (but an approximate) match in a reasonable response time

Traditional recruiting systems do not include such a kind of knowledge base and appropriate algorithms for exploiting it, and mainly due to this reason, their decisions are far away from the behavior of an expert recruiter. Therefore, it is supposed that the contribution of knowledge management can notably improve the traditional job recruitment processes.

\subsection{The matching process}

In this context, semantic matching is a computational process whereby two entities in a job offer and applicant profile respectively are assigned a score based on the likeness of their meaning. Traditionally, the way to compute the degree of correspondence between entities has been addressed from two different perspectives: using semantic similarity measures and semantic relatedness measures. However, recent works in this field have clearly defined the scope of each of them (Batet, 2010). 
Firstly, semantic similarity is used when determining the taxonomic proximity between objects. For example, automobile and car are similar because the relation between both terms can be defined by means of a taxonomic relation. Secondly, the more general concept of semantic relatedness considers taxonomic and relational proximity. For example, blood and hospital are not completely similar, but it is still possible to define a naive relation between them because both belong to the world of healthcare. Due to the impact of measuring similarity in modern computer science, most of existing works are focused on semantic similarity, but many of proposed ideas are also applicable to the computation of relatedness.

In our case, the problem to face is much more complex since it does not involve the matching of two individual entities only, but a job offer and many applicant profiles. This can be achieved by computing a set of semantic correspondences between individual entities belonging to the job offer and each of the applicant profiles. A set of semantic correspondences between these objects is often called an alignment.

Therefore, when matching a job offer and an applicant profile, the challenge scientists try to address consists of finding an appropriate semantic matching function leading to a high quality alignment. Quality here is measured by means of a function that associates an alignment and an ideal alignment to two real numbers stating the precision and recall of the first in relation to the second one.

Precision represents the notion of accuracy, that it is to say, states the fraction of retrieved correspondences that are relevant for the matching task ( 0 stands for no relevant correspondences, and 1 for all correspondences are relevant). Meanwhile, recall represents the notion of completeness, thus, the fraction of relevant correspondences that were retrieved (0 stands for not retrieved correspondences, and 1 for all relevant correspondences were retrieved). 
Another important factor that semantic matching allows taking into account is overqualification ${ }^{1}$. Notion of overqualification reflects candidates which fully fulfill all job requirements, but which can still be unsuitable for a job, because their background is too advanced. Overqualification can also be measured using the relations of the HR-Knowledge Base.

The potential of this kind of techniques is that it can support HRM when leading to a more quickly and easily cut through massive volumes of potential candidate information, but without giving up the way human experts take decisions in the real world.

\subsection{Matching learning from solved cases}

Approaches using knowledge management aim at improving a knowledge-based system by adapting both the background knowledge and the algorithms. This can be done by means of automatically learning from past solved cases.

The function finally used to recommend candidates to employers (and vice versa) is a combination of the candidate fitness function and the job interest function. The form of this combination can be learned from solved cases using machine learning methods, including genetic algorithms, neural networks, and so on (Faliagka et al., 2012). Training data can be matching assessments from HR experts and user feedback.

Successfully learning from past solved cases is very important for the performance of a HRM system. Traditionally, there have been some heuristic approaches to do that, for example counting the edges between concepts to assess similarity or set-theoretic ones which compute with union of all the concepts above the given ones. But these methods are all heuristic and do not

${ }^{1}$ Disclaimer: Please note that authors think that the concept of overqualification is unfair. However, this concept has been included in this manuscript since it is a major concern for many players in the HR domain. 
consider learning from real world empirical matchings as statistical machine learning approaches do.

Therefore, although some kind of machine learning techniques has to be applied, the resulting matching should be still knowledge-based so that can be interpreted accordingly. In this way, it is possible to get the advantages of the two methodologies, the interpretability of knowledge-based solutions and the adaptability of statistical machine learning systems. This is in contrast to pure statistical methods such as bag-of-word approaches and similar methods, which are hard to interpret.

On the other hand, if instances are described with a controlled vocabulary, and additionally there are terms which are missing in the HR-Knowledge Base. The solution space is parameterized by both the form of the knowledge base and the form of the function. This means that in order to improve the matching process, it is necessary to adjust objects, the knowledge base as well as the matching function in order to identify what is the meaning of these apparently missing terms.

\subsection{Automatic enrichment of HR-Knowledge Bases}

Automatic enrichment of HR-Knowledge Bases could be considered a problem analogous to Ontology Learning (Shamsfard \& Barforoush, 2003). However, there is a difference since enrichment processes do not try to build a knowledge base from scratch, rather to refine (enrich) an existing one.

Several surveys from the literature deal with this problem; Shamsfard \& Barforoush which present a complete framework that classifies software and techniques for building ontologies (Shamsfard \& Barforoush, 2004), and Cimiano et al. who provide a comprehensive tutorial on learning ontology from text (Cimiano et al., 2003). 
Figure 3 shows an example of an admissible enrichment for a HR-Knowledge Base. Let us suppose that we have initially a set of concepts and relationships between them representing some knowledge about the Business Administration field. Completing Administrative Functions is part of this field, and Staffing, Controlling and Team Leading are also some kinds of Administrative Functions. Now, the system realizes that many candidates from the applicant's database are including Organizing as a competency. In that case, it is necessary to reorganize the knowledge base to place in successfully this new concept. The algorithms should be able to detect that Organizing is a superior concept of Team Leading (since it probably includes other many aspects such as Team Creation, Training, and so on). Therefore this approach has to be able to automatically reorganize this part of our HR-Knowledge Base accordingly.

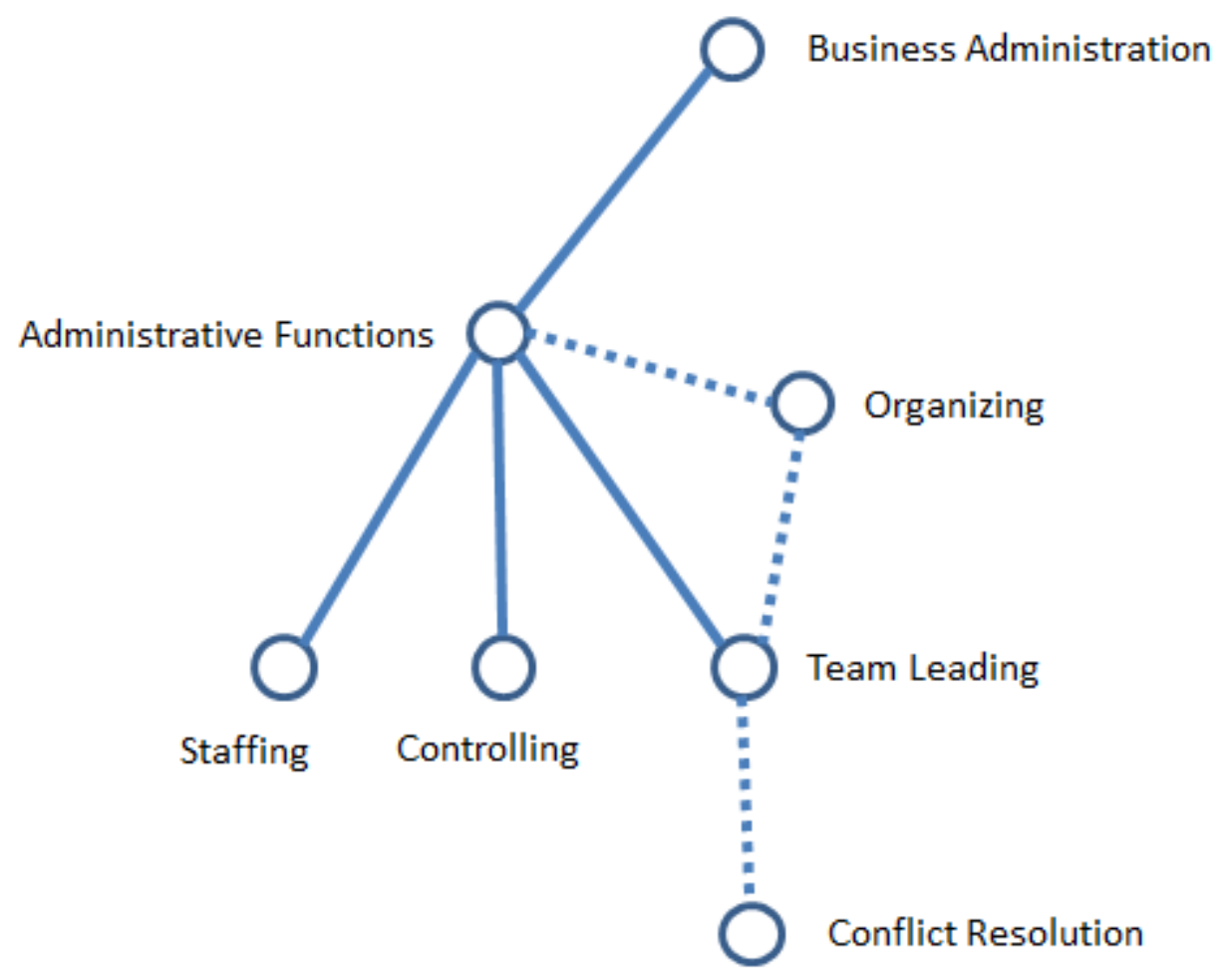

Figure 3. Example of consistent enrichment of a lattice that represents features for modeling industrial knowledge

At the same time, let us suppose that the system also realizes that many candidates from the applicant's database claim to be experts on Conflict Resolution. Once again, this system has to be able to recognize that solving conflicts that may arise in a team is one of the common tasks that a team leader 
should address. Therefore, the HRM system should be able to automatically restructure our set of concepts and relationships.

FCA (Ganter \& Wille, 1999) is used to produce a lattice (set of concepts and relationships between them) that is then converted into a special kind of partial order constituting a concept hierarchy (Cimiano et al., 2004). FCA can be applied to generate information context from a tentative domain specific scientific text, corpus or database which is then mapped to a formal ontology (Jia, Newman \& Tianfield 2007). This paradigm can be applied in many different realms like psychology, sociology, anthropology, linguistics, computer science, mathematics and industrial engineering (Kuznetsov \& Poelmans, 2013). In this scenario, it is necessary to generate content from a database of job applicants in order to build and/or enrich HR-Knowledge Bases concerning human resources. This approach is appropriate since a semi-automatic method to ontology design, with a set of rules mapping a FCA lattice to a rule language is presented with successful results in (Haav 2004). One of the advantages of using FCA for enriching knowledge bases modeling concepts, attributes and relationships is that it produces concept lattices which allow a concept to have more than a single super concept.

Moreover, the FCA paradigm can be also used for the extraction of frequent items from databases, that is to say, sets of attributes occurring together with a certain frequency (Kumar, 2012). This fact allows us to identify association rules emphasizing correlations between sets of attributes with a given confidence (Bal et al., 2011). The search for frequent items and association rules extraction are well-known symbolic data mining methods. These processes usually produce a large number of items and rules, leading to the associated problems of mining the sets of extracted items and rules. According to $\mathrm{Bal}$ et al, association rules with $100 \%$ confidence are called implication (Bal et al., 2011).

The major idea consists of using these association rules for enriching the knowledge base. Bal et al. demonstrated that this is possible since some works have shown us that it is possible to discover a lot of knowledge from databases of job applicants in the form of association rules (Bal et al., 2011). In this way, for example, if a major number of applicant profiles show that studying Finance 
leads to the acquisition of Accountability skills, then we can enrich our knowledge base modeling some kind of industrial knowledge by means of these new knowledge. Obviously, the degree of confidence and support for each of the new knowledge discovered has to be appropriately managed, so that the HRKnowledge Base can be enriched but without giving up some certain quality criteria concerning accuracy.

\subsection{Adaptation of matching methods to allow Top-K queries}

In many cases, it is interesting to explore the database of applicant's profiles to get an ordered list of top candidates for a given job offer. One example of query could consist of retrieving the most experienced programmers when using fourthgeneration programming languages. In cases of this kind, it is necessary to get some additional information from a HR-Knowledge Base since it is not usual that people specify this kind of information in their CVs.

The major problem here is that this kind of queries is very time consuming in general, since it is necessary to compute the fitness for the match between the given job offer and each of the applicant's profiles contained in the database. This means that if the amount of profiles to process is high, the response can be delayed for a long period of time.

However, being able to process this kind of queries is a crucial requirement in environments that involve massive amounts of data. It is necessary to decide many aspects for this kind of queries including query models, data access methods, implementation levels, data and query certainty. All of these features have to be analyzed in order to determine which is the best configuration for a HRM system of these characteristics.

This kind of problem can be appropriately addressed by means of the Top-K query model (Soliman et al., 2010), which is a computational paradigm which allows to model a balance between accuracy of the results and the time necessary to reach them. 
The Top-K query model tries to overcome the problem concerning queries over structured and semi-structured data. In this model, queries try to identify the appropriate matches for a given request. This query model is not appropriate for database applications and scenarios where queries are expressing user preferences and not boolean constraints. Therefore, the kind of queries is best served with a ranked list of the best matching objects, for some definition of degree of match. For this reason the Top-K query model was introduced (Chakrabarti et al., 2004).

In the scope of HRM, recruiters are often interested in the most important (TopK) query answers in the potentially huge answer space formed by many thousands of applicant's profiles. In this way, it is possible to create a ranking with the most promising candidates who meet the requirements for a job offer (or simply a specific combination of skills or competencies) within a tunable trade-off between the accuracy of the results and the response time.

This problem has traditionally been addressed by using high-dimensional indexes built on individual data features, and a nearest-neighbor scan operator on top of these indexes. A database system that supports matching ranks objects depending on how well they match the query example. In many situations, it is necessary to consider some kind of partial matchers (i.e. matchers which can perform only some key operations, and therefore, are faster) so that the matching process can be accelerated. Otherwise, a database containing many thousands of applicant's profiles could make the search process unfeasible.

\section{Conclusions \& Future Research Lines}

In this work, we have described the major advances in the e-Recruitment field through the use of advanced knowledge management techniques. There are a number of major advantages over the state-of-the-art in this field that can be summarized in the following five points: 
1. Knowledge management allows job recruiters to reduce the costs and time to find relevant matches between job offers and applicant profiles. This fact is strongly positive in organizations with a high volume of hiring needs. The reason is that, in complicated labor markets or regions allowing free movement of workers, factors like cost or time are becoming critical.

2. Knowledge management techniques for semantic matching, enriching of HR-Knowledge Bases and Top-K querying can help players from the HR industry to go beyond identical lexical matching of job offers and applicant profiles. This represents a great advantage over the state-of-the-art since give more opportunities to the good job candidates, but also allows job recruiters to identify potential talent which otherwise may remain blurred among such a plethora of applicant's profiles.

3. Knowledge management can help to eliminate the need for job recruiters to have deep and specialized knowledge within an industry or skill set. This is mainly due to a HR-Knowledge Base is able to model knowledge from a lot of industrial domains. Then this knowledge can be used as a support when performing matching process so that the results can be very similar to those produced by an expert person from that field.

4. Techniques for taking into account the Top-K query model can help to reduce or even eliminate the time spent on initial preprocessing of job applicant's profiles. This is mainly due to this kind of query allows job recruiters to formulate search expressions based on user preferences which are going to deliver the most relevant results in a reasonable response time. This functionality also represents an advantage for recruiting teams with low search capability.

5. Overviewed techniques allow to leveling the odds for those job applicants with less experience or ability when preparing their resumes. Within an automatic approach, a computational algorithm will perform the matching process automatically. The result from this matching process is 
independent of the way the curriculum is presented. Therefore, this kind of techniques helps to promote equal opportunities.

Future work should focus on the impact on improving the functionality of existing HR-Knowledge Bases like European Dictionary of Skills and Competences ${ }^{2}$ (DISCO) by providing tools for permanent improvement and refinement. Moreover, using appropriate knowledge management techniques would also allow a tight integration and fusion of different existing HR-Knowledge Bases. Fusing and linking different HR-Knowledge Bases should enable the creation of novel and enriched HR-Knowledge Bases to serve as base for numerous applications built upon.

\section{Acknowledgements}

We would like to thank the anonymous reviewers for their help to improve this work.

\section{References}

Bal, M, Y Bal and A Ustundag (2011). Knowledge representation and discovery using formal concept analysis: An HRM application. In Proc. World Conference on Engineering, pp. 1068-1073.

Batet, M, D Sánchez and A Valls (2011). An ontology-based measure to compute semantic similarity in biomedicine. Journal of Biomedical Informatics, 44(1), 118125.

Bizer, C, R Heese, M Mochol, R Oldakowski, R Tolksdorf and R Eckstein (2005). The impact of semantic web technologies on job recruitment processes. Proc of Wirtschaftsinformatik, pp. 1367-1381, Physica-Verlag HD.

\footnotetext{
${ }^{2}$ http://disco-tools.eu/disco2_portal/
} 
Bradley, K and B Smyth (2003). Personalized information ordering: a case study in online recruitment. Knowl.-Based Syst, 16(5-6), 269-275.

Chakrabarti, K, M Ortega-Binderberger, S Mehrotra and K Porkaew (2004). Evaluating refined queries in top-k retrieval systems. IEEE Transactions on Knowledge and Data Engineering, 16(2), 256-270.

Cimiano, P, A Hotho, G Stumme and J Tane (2004). Conceptual knowledge processing with formal concept analysis and ontologies. Proc. of Concept Lattices, pp. 189-207, Springer Berlin Heidelberg.

Colucci, S, TD Noia, ED Sciascio, FM Donini, M Mongiello and M Mottola (2003). A formal approach to ontology-based semantic match of skills descriptions. $J$. UCS, 9(12), 1437-1454.

Daramola JO, O Oladipupo, AG Musa (2010). A fuzzy expert system (FES) tool for online personnel recruitments. IJBIS, 6(4), 444-462.

Faliagka, E, K Ramantas, A Tsakalidis and G Tzimas (2012). Application of machine learning algorithms to an online recruitment system. In ICIW 2012, The Seventh International Conference on Internet and Web Applications and Services, pp. 215-220.

Faliagka, E, I Karydis, M Rigou, S Sioutas, A Tsakalidis and G Tzimas (2012). Taxonomy Development and Its Impact on a Self-learning e-Recruitment System. In Artificial Intelligence Applications and Innovations, pp. 164-174, Springer Berlin Heidelberg.

Faliagka E, A Tsakalidis, G Tzimas (2012). An Integrated E-Recruitment System for Automated Personality Mining and Applicant Ranking. Internet Research, 22(5): 551-568. 
Färber, F, T Weitzel and T Keim (2003). An automated recommendation approach to selection in personnel recruitment. In Proceedings of the Ninth Americas Conference on Information Systems (pp. 4-6).

Ganter, B, R Wille and C Franzke (1997). Formal concept analysis: mathematical foundations. Springer-Verlag New York, Inc.

García-Sánchez, F, R Martínez-Béjar, L Contreras, JT Fernández-Breis and D Castellanos-Nieves (2006). An ontology-based intelligent system for recruitment. Expert Systems with Applications, 31(2), 248-263.

Haav, HM (2004). A semi-automatic method to ontology design by using FCA. In Proceedings of CLA (Vol. 4).

Jia, H, J Newman and H Tianfield (2009). A new Formal Concept Analysis based learning approach to Ontology building. In Metadata and Semantics, pp. 433-444. Springer US.

Kumar, ChA (2012). Fuzzy Clustering-Based Formal Concept Analysis for Association Rules Mining. Applied Artificial Intelligence, 26(3), 274-301.

Kuznetsov, SO and JO Poelmans (2013). Knowledge representation and processing with formal concept analysis, Data Mining and Knowledge Discovery 3(3), 200-215.

Khosla, R, T Goonesekera and MT Chu (2009). Separating the wheat from the chaff: An intelligent sales recruitment and benchmarking system, Expert Syst. Appl. 36(2), 3017-3027.

Senthil Kumaran, V and A Sankar (2013). Towards an automated system for intelligent screening of candidates for recruitment using ontology mapping (EXPERT). IJMSO, 8(1), 56-64. 
Malinowski, J, T Keim, O Wendt, T Weitzel (2006). Matching people and jobs: A bilateral recommendation approach. In System Sciences, 2006. HICSS'06. Proceedings of the 39th Annual Hawaii International Conference on (Vol. 6, pp. 137c-137c). IEEE.

Martinez-Cruz, C, IJ Blanco, and MA Vila (2012). Ontologies versus relational databases: are they so different? A comparison, Artificial Intelligence Review, 38(4), 271-290.

Mochol, M, H Wache and L Nixon (2007). Improving the accuracy of job search with semantic techniques. In Business Information Systems, pp. 301-313, Springer Berlin Heidelberg.

Shamsfard, M and AA Barforoush (2003). The state of the art in ontology learning: a framework for comparison, The Knowledge Engineering Review, 18(4), 293-316.

Shamsfard, M and AA Barforoush (2004). Learning ontologies from natural language texts. International Journal of Human-Computer Studies, 60(1), 17-63.

Smyth, B, K Bradley and R Rafter (2002). Personalization techniques for online recruitment services, Commun. AC, M 45(5), 39-40.

Soliman, MA, IF Ilyas and S Ben-David (2010). Supporting ranking queries on uncertain and incomplete data, The VLDB Journal, 19(4), 477-501.

Wen, K, Y Zeng, R Li, J Lin (2012). Modeling semantic information in engineering applications: a review, Artif Intell Rev, 37(2), 97-117. 